\title{
Corneal higher-order aberrations of the anterior surface, posterior surface, and total cornea after small incision lenticule extraction (SMILE): high myopia versus mild to moderate myopia
}

Hong-Ying Jin*, Ting Wan, Xiao-Ning Yu, Fang Wu and Ke Yao

\begin{abstract}
Background: To investigate corneal higher-order aberrations (HOAs) of the anterior surface, posterior surface, and total cornea after small incision lenticule extraction (SMILE) in high myopic and mild to moderate myopic patients.

Methods: This retrospective study included 197 eyes (101 patients) undergoing SMILE surgery. According to the preoperative spherical equivalent (SE), treated eyes were divided into two groups: a high myopic group (more than - 6.0 D, Group H) and a mild to moderate myopic group (less than - 6.0 D, Group M). Corneal HOAs of the anterior surface, posterior surface, and total cornea were measured using a Scheimpflug camera preoperatively and 3 months postoperatively. Pearson's correlation analysis was conducted to determine relationships between corneal aberrations and the SE.
\end{abstract}

Results: There were no significant differences in third-order to eight-order aberrations (RMS HOAs) of the anterior surface, posterior surface, and total corneal between the two groups before SMILE surgery. However, after SMILE, anterior and total corneal HOAs, especially vertical coma and spherical aberrations, significantly increased in both groups $(p<0.0167)$, whereas posterior corneal HOAs remained relatively stable $(p>0.0167)$. The induction of HOAs was significantly greater in Group $H$ than Group M postoperatively $(p<0.0167)$. Changes in anterior surface and total corneal HOAs, especially vertical coma and spherical aberrations, were related to the SE $(p<0.05)$.

Conclusions: Anterior and total corneal HOAs, particularly vertical coma and spherical aberrations, significantly increased after SMILE in both groups, whereas posterior corneal HOAs remained stable. Aberration changes were related to SE.

Trial registration: Retrospectively registered. ChiCTR-ORC-17011040. Registered 1 April 2017. Name of registry: The observation of clinical results after corneal refractive surgery. Data of enrolment of the first participant to the trial: 15 December 2016.

Keywords: SMILE, Higher-order aberrations, Posterior cornea

\footnotetext{
* Correspondence: hongyingj@zju.edu.cn

Eye Center, 2nd Affiliated Hospital, School of Medicine, Zhejiang University,

Hangzhou 310009, China
}

(c) The Author(s). 2018 Open Access This article is distributed under the terms of the Creative Commons Attribution 4.0 International License (http://creativecommons.org/licenses/by/4.0/), which permits unrestricted use, distribution, and reproduction in any medium, provided you give appropriate credit to the original author(s) and the source, provide a link to the Creative Commons license, and indicate if changes were made. The Creative Commons Public Domain Dedication waiver (http://creativecommons.org/publicdomain/zero/1.0/) applies to the data made available in this article, unless otherwise stated. 


\section{Background}

Visual acuity is the most commonly used parameter for assessments of overall visual function in the clinical setting. However, under dim light conditions or low contrast sensitivity conditions, visual acuity does not truly reflect a patient's subjective vision function [1], especially after corneal refractive surgeries. Higher-order aberrations (HOAs) increase after successful refractive surgery and influence the vision quality of patients. Measurements of changes in wavefront aberrations are used to evaluate the impact of refractive surgery on vision quality [1]. Previous studies indicated that posterior corneal HOAs influenced corneal biomechanical responses and provided valuable information in determining the cause of poor vision quality after corneal refractive surgery $[2,3]$.

Small incision lenticule extraction (SMILE) surgery is a newly developed corneal refractive technique, which is less invasive than LASIK (Laser-assisted in situ keratomileusis) and FS-LASIK (femtosecond laser-assisted LASIK), with only a small incision required $[4,5]$. Recent studies suggested that the SMILE procedure provided excellent clinical outcomes, considering its safety, efficacy, predictability, and postoperative ocular surface health [6-8]. Therefore, SMILE is considered to be a good choice for refractive surgery. Previous studies of changes of corneal HOAs after the SMILE procedure focused mainly on anterior corneal HOAs or total corneal HOAs [8-11]. Only a few studies have compared anterior, posterior, and total corneal HOAs following different refractive surgery procedures $[2,3,12]$. The potential effect of the SMILE procedure on posterior corneal HOAs is not well understood. In this study, we compared HOAs of the anterior surface, posterior surface, and total cornea after SMILE surgery for high myopia and mild to moderate myopia.

\section{Material and methods Subjects}

This is a retrospective study, which recruited 197 eyes (101 patients). All patients aged between 18 and 47 years underwent SMILE surgery at the Department of Ophthalmology, Second Affiliated Hospital, College of Medicine, Zhejiang University from December 2016 to May 2017 were included in this study. This research followed the tenets of the Declaration of Helsinki was approved by the Institutional review board (No: 2017017). Informed written consent was obtained from the all subjects. The inclusion criteria were aged $\geq 18$ years, myopic spherical correction $<-10.00$ diopters, no ocular or systemic diseases, and stable refraction for at least one year. The exclusion criteria were the presence of active ocular disease or a history of ocular surgery and trauma. Patients were instructed to stop wearing soft contact lenses for at least 1 week prior to the surgery. The cases were divided into two groups according to the degree of preoperative spherical equivalent (SE): a high myopic group (more than - 6.0 D,Group $\mathrm{H}$ ) and a mild to moderate myopic group (less than $-6.0 \mathrm{D}$, Group $\mathrm{M}$ ).

\section{Surgical technique}

All surgeries were performed by the same experienced surgeon (H.Y.J.). A VisuMax femtosecond laser system (Carl Zeiss Meditec AG, Jena, Germany) was used for surgical refractive corrections in all patients, with a repetition rate of $500 \mathrm{kHz}$ and pulse energy of $155 \mathrm{~nJ}$. The stroma cap was set at thickness of $120 \mu \mathrm{m}$, diameter of $7.5-7.6 \mathrm{~mm}$. Prior to the initiation of suction, the patients were instructed to fixate on a target light. Four cleavage planes were created, including anterior and posterior surfaces of the refractive lenticule and vertical edge of the refractive lenticule. A single side-cut incision (width of $2 \mathrm{~mm}$ ) was made at an angle of $120^{\circ}$. The lenticule was removed using a forceps. The target refraction was emmetropia. After surgery, all the patients received a topical antibiotic for 7 days and a topical steroid for 2 weeks. Artificial tears were used for more than 4 weeks.

\section{Measurement of corneal aberrations}

Corneal aberrations of the anterior surface, posterior surface, and total cornea were measured by a rotating Scheimpflug Camera (Pentacam HR; Oculus, Wetzlar, Germany). The Pentacam HR is a noninvasive and reproducible diagnostic method. The measurements were made in a dark room. To minimize the potential effect of tear film on corneal imaging, the patients were instructed to remain fixated on a target light immediately after blinking. The Pentacam HR camera then started rotating and scanning the cornea. Only measurements marked as "OK" quality were considered valid. Corneal aberrations of the anterior surface, posterior surface, and total cornea were analyzed over a $6.0-\mathrm{mm}$ central diameter preoperatively, 1 and 3 months postoperatively. Root mean square (RMS) values of higher-order aberrations (HOAs) (third-order to eight-order), including coma, trefoil, quadrafoil, and secondary astigmatism, were calculated.

\section{Statistical analysis}

Statistical analyses were performed using SPSS software, ver. 18 (SPSS, Chicago, IL, USA). The KolmogorovSmirnov test was used to test for normality. An independent-sample $t$ test was conducted for comparisons between Group H and Group M. A paired-sample $t$ test was performed for preoperative and postoperative comparisons. The Bonferroni correction for multiple testing was used to reduce the rate of type I error. In addition, Pearson's correlation analysis was performed to determine relationships between the preoperative spherical equivalent (SE) and corneal aberrations. All values are given as the 
mean \pm standard deviation. $P<0.05$ was considered statistically significant, otherwise indicated.

\section{Results}

All 101 patients attended the 1-month, and 3-month follow-up examinations. Group $\mathrm{H}$ and Group $\mathrm{M}$ comprised 65 and 132 eyes, respectively. Preoperative characteristics of both groups are listed in Table 1. There were no statistically significant differences between the two groups as regards the patient's age, preoperative SE, spherical diopter, cylindrical diopter, central corneal thickness(CCT), intraocular pressure(IOP), and mean corneal power, making it possible to compare corneal HOAs without confounders $(p>0.05)$.

\section{Comparison of surgically induced third-order to eight-} order aberrations (RMS HOAs) after SMILE in group $\mathrm{H}$ and group $M$

As shown by RMS values, third-order to eight-order aberrations of the anterior corneal surface (RMS-HOA-CF) and total cornea (RMS-HOA-cornea) significantly increased after SMILE surgery in Group $\mathrm{H}$ and Group $\mathrm{M}$ ( $p<0.0167)$ (Fig. 1a and c). According to RMS values, posterior corneal HOAs (RMS-HOA-CB) slightly increased, but remained relatively stable in both groups $(p>0.0167)$ (Fig. 1b).

\section{Comparison of preoperative HOAs versus surgically induced HOAs (third-order to eight-order, RMS HOAs) between group $\mathrm{H}$ and group $\mathrm{M}$}

As shown by RMS values, there were no significant differences in HOAs between Group $\mathrm{H}$ and Group M preoperatively $(p>0.0167)$. However, there were significant differences in HOAs in anterior cornea and total cornea between the two groups 1 and 3 months after surgery $(p<0.0167)$. There was no significant difference in HOAs of posterior cornea surface between the two groups after the surgery $(p>0.0167)$ (Table 2$)$.

\section{Comparison of surgically induced anterior corneal surface HOAs in group $\mathrm{H}$ and group $\mathrm{M}$}

There were no significant differences in coma and spherical aberrations of the anterior surface between the two groups preoperatively $(p>0.0167)$. However, there was a significant increase in vertical coma $\left(\mathrm{Z}^{3,-1}\right)$ and spherical aberrations $\left(Z^{4,}{ }^{0}\right)$ in the two groups 1 and 3 months postoperatively $(p<0.0167)$. More coma and spherical aberrations of the anterior corneal surface were induced in Group $\mathrm{H}$ than Group M postoperatively $(p<0.0167)$ (Fig. 2).

\section{Comparison of surgically induced posterior corneal surface HOAs in group $\mathrm{H}$ and group $\mathrm{M}$}

There was no significant difference in spherical aberrations $\left(Z^{4,}{ }^{0}\right)$ of the posterior cornea between the two groups preoperatively or postoperatively $(p>0.0167)$. There was also no significant increased in spherical aberration in the two groups after smile surgery $(p>0.0167)$. There was no significant differences between-group in coma aberrations preoperatively $(p>0.0167)$. However, there were significant changes in vertical coma $\left(\mathrm{z}^{3,-1}\right)$ and vertical trefoil $\left(Z^{3,-3}\right)$ after SMILE surgery in the two groups $(p<0.167)$. In addition, there was significant difference in vertical coma between the two groups after surgery $(p<0.167)$ (Fig. 3).

\section{Comparison of surgically induced total corneal HOAs in group $\mathrm{H}$ and group $\mathrm{M}$}

There were no significant differences in total corneal HOAs (coma and spherical aberrations) between the two groups preoperatively $(p>0.0167)$. In contrast, there were significant differences in vertical coma $\left(\mathrm{Z}^{3,-1}\right)$ and

Table 1 Demographic and preoperative patient information (mean \pm SD)

\begin{tabular}{|c|c|c|c|c|}
\hline Parameter & Group H & Group M & $t$ & $p$ \\
\hline Eye $(n)$ & 65 & 132 & & \\
\hline $\operatorname{Sex}(M / F)$ & $31 / 34$ & $77 / 56$ & & \\
\hline Age (y) & $24.46 \pm 7.34$ (18 to 47$)$ & $23.84 \pm 5.92(18$ to 47$)$ & 0.64 & 0.52 \\
\hline IOP (mmHg) & $15.80 \pm 2.12(11$ to 23$)$ & $15.48 \pm 2.36(10$ to 23$)$ & 0.93 & 0.36 \\
\hline $\mathrm{CCT}(\mu \mathrm{m})$ & $552.58 \pm 25.61$ (502 to 616$)$ & $541.52 \pm 28.03$ (482 to 627$)$ & 1.66 & 0.10 \\
\hline Mean corneal power (D) & $43.16 \pm 1.42$ (40.60 to 46.55$)$ & $43.24 \pm 1.25$ (41.25 to 45.95$)$ & -0.42 & 0.67 \\
\hline SE (D) & $-7.32 \pm 0.99(-6.00$ to -9.88$)$ & $-4.45 \pm 1.01(-1.13$ to -5.88$)$ & -18.85 & $0.00^{*}$ \\
\hline Sphere (D) & $-6.94 \pm 1.00(-5.00$ to -9.75$)$ & $-4.13 \pm 1.00(-0.75$ to -5.75$)$ & -18.60 & 0.00 \\
\hline Cylinder (D) & $-0.76 \pm 0.68$ (0 to -3.00$)$ & $-0.64 \pm 0.50(0$ to -2.75$)$ & -1.35 & 0.18 \\
\hline Lenticule thickness ( $\mu \mathrm{m})$ & $128.48 \pm 10.27$ (109 to 148$)$ & $92.23 \pm 15.53$ (50 to 120$)$ & 19.51 & $0.00^{*}$ \\
\hline Lenticule diameter (mm) & $6.52 \pm 0.14$ (6.1 to 6.6$)$ & $6.58 \pm 0.05$ (6.1 to 6.6$)$ & -5.84 & $0.00^{*}$ \\
\hline
\end{tabular}

$S D$ standard deviation, $D$ diopters, SE spherical equivalent, $C C T$ central corneal thickness, IOP intraocular pressure, Group $H$ high myopia group, Group $M$ mild to moderate myopia group.* $p<0.05$ 

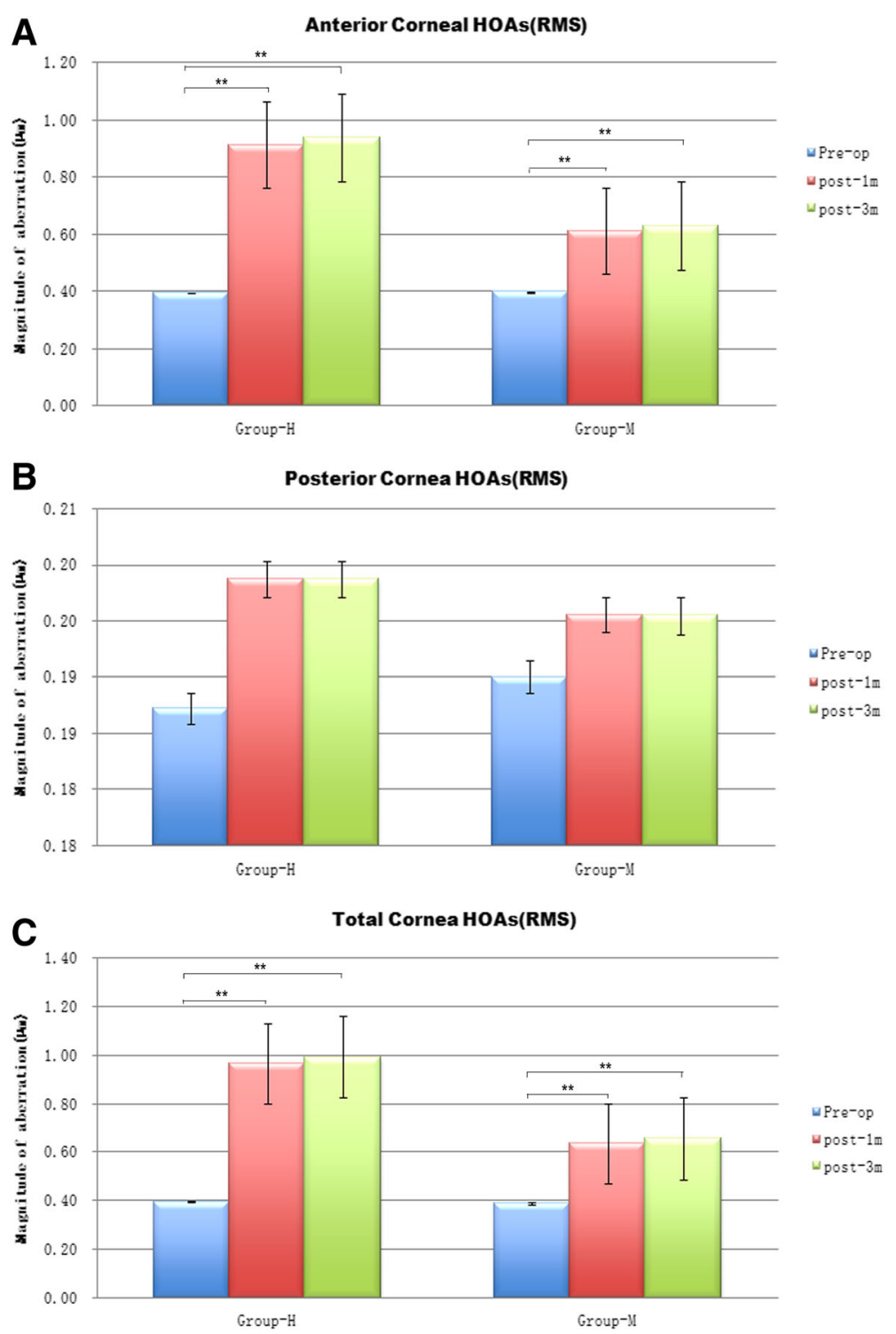

Fig. 1 Third-order to eight-order higher-order aberrations (RMS HOAs) of the cornea preoperatively and 1 and 3 months postoperatively in Group $\mathrm{H}$ and Group M. a Anterior corneal surface (RMS-HOA-CF, root mean square -HOA- cornea front); b Posterior corneal surface (RMS-HOA-CB, root mean square-HOA- cornea back); c Total cornea (RMS-HOA-cornea, root mean square- HOA- cornea). ${ }^{*} p<0.0167$ (the modified Bonferroni correction, 0.05/3 tests)

spherical aberrations $\left(Z^{4,}{ }^{0}\right)$ between the two groups 1 and 3 months postoperatively $(p<0.0167)$. More total corneal coma and spherical aberrations were induced in Group $\mathrm{H}$ than Group M postoperatively $(p<0.0167)$ (Fig. 4).

\section{Correlations between the preoperative SE and surgically induced corneal HOAs}

Correlations between the preoperative SE and surgically induced corneal HOAs are shown in Table 3. The SE was significantly correlated with the induction of HOAs (third-order to eight-order, RMS HOAs) of the anterior surface and total cornea, especially vertical coma and spherical aberrations after SMILE surgery $(p<0.05)$ (Table 3).

\section{Discussion}

This study is the first to compare corneal HOAs of the anterior surface, posterior surface, and total cornea in high and mild to moderate myopic eyes after SMILE surgery. The results indicated that HOAs of the anterior cornea surface and total cornea significantly increased after SMILE surgery in both groups. However, HOAs of the posterior corneal surface remained relatively 
Table 2 Comparison of higher-order aberrations (HOAs) between the two groups preoperatively and postoperatively 1 and 3 months (mean \pm SD)

\begin{tabular}{|c|c|c|c|c|c|c|c|c|c|}
\hline \multirow{2}{*}{$\begin{array}{l}\text { Time } \\
\text { Group }\end{array}$} & \multicolumn{3}{|l|}{ Preop. } & \multicolumn{2}{|c|}{ Postop. 1 month } & \multirow[b]{2}{*}{ p } & \multicolumn{3}{|c|}{ Postop. 3 months } \\
\hline & $\begin{array}{l}\text { Group H } \\
(n=65)\end{array}$ & $\begin{array}{l}\text { oup M } \\
=132 \text { ) }\end{array}$ & $p$ & $\begin{array}{l}\text { Group H } \\
(n=65)\end{array}$ & $\begin{array}{r}M \\
32)\end{array}$ & & & $\begin{array}{l}\text { roup } M \\
=132)\end{array}$ & $P$ \\
\hline MS HOA (CF) & $0.39 \pm 0.08$ & $0.40 \pm 0.11$ & 0.754 & $0.91 \pm 0.28$ & $0.61 \pm 0.18$ & $0.000^{*}$ & $0.94 \pm 0.26$ & $0.63 \pm 0.19$ & 0.00 \\
\hline MS HOA (CB) & 3 & 03 & 5 & 03 & 03 & 0.219 & $0.19 \pm 0.03$ & .03 & 0.640 \\
\hline MS HOA (cornea) & $0.40 \pm 0.09$ & $0.39 \pm 0.12$ & 0.710 & $0.97 \pm 0.30$ & $0.64 \pm 0.19$ & 0.000 & $0.99 \pm 0.28$ & $0.66 \pm 0.20$ & 0.000 \\
\hline blique trefoil $Z^{3,3}$ (CF) & 07 & $-001+0$ & 0.294 & $0.01 \pm 0.10$ & $-0 \Omega 1+0$ & 0.486 & $0.03 \pm 0.11$ & $0.01 \pm 0.11$ & 0.37 \\
\hline orizontal coma Z Z & $-0.01 \pm 0.15$ & $-0.03 \pm 0.15$ & 0.304 & $0.01 \pm 0.37$ & $-0.04 \pm 0.25$ & 0.434 & $-0.03 \pm 0.38$ & $-0.05 \pm 0.26$ & 0.996 \\
\hline rrtical coma $Z^{3,-1}$ (CF) & $-0.03 \pm 0.19$ & $0.01 \pm 0.18$ & 0.276 & $-0.60 \pm 0.31$ & $-0.34 \pm 0.23$ & $0.000^{*}$ & $-0.62 \pm 0.29$ & $-0.32 \pm 0.26$ & 0.000 \\
\hline ertical trefoil $Z^{3,-3}(C F)$ & $-0.04 \pm 0.10$ & $-0.05 \pm 0.11$ & 0.666 & $-0.03 \pm 0.13$ & $-0.02 \pm 0.11$ & 0.988 & $-0.04 \pm 0.12$ & $-0.03 \pm 0.13$ & 0.661 \\
\hline spherical aberration $Z^{4,0}$ (CF) & $0.24 \pm 0.07$ & $0.26 \pm 0.07$ & 0.188 & $0.44 \pm 0.14$ & $0.31 \pm 0.11$ & $0.000^{*}$ & $0.45 \pm 0.13$ & $0.30 \pm 0.11$ & 0.00 \\
\hline blique trefoil $Z^{3,3}(\mathrm{CB})$ & $-0.01 \pm 0.05$ & \pm 0.04 & 0.619 & $0.00 \pm 0.05$ & $-0.01 \pm 0.04$ & 0.490 & $-0.01 \pm 0.04$ & 05 & 0.895 \\
\hline orizontal coma $Z^{3,1}$ (CB) & $0.00 \pm 0.02$ & $0.00 \pm 0.02$ & 0.408 & $-0.01 \pm 0.03$ & $0.00 \pm 0.03$ & 0.180 & $-0.01 \pm 0.03$ & $0.00 \pm 0.03$ & 0.461 \\
\hline Vertical coma $Z^{3,-1}$ (CB) & $-0.02 \pm 0.03$ & $-0.03 \pm 0.04$ & 0.025 & $0.01 \pm 0.04$ & $-0.01 \pm 0.04$ & $0.000^{*}$ & $0.01 \pm 0.03$ & $-0.01 \pm 0.04$ & 0.00 \\
\hline Vertical trefoil $Z^{3,-3}(C B)$ & $-0.02 \pm 0.05$ & $-0.02 \pm 0.05$ & 0.220 & $-0.05 \pm 0.05$ & $-0.04 \pm 0.06$ & 0.249 & $-0.04 \pm 0.05$ & $-0.04 \pm 0.05$ & 0.470 \\
\hline Spherical aberration $Z^{4,0}(C B)$ & $-0.15 \pm 0.03$ & $-0.16 \pm 0.03$ & 0.306 & $-0.16 \pm 0.03$ & $-0.16 \pm 0.03$ & 0.021 & $-0.16 \pm 0.03$ & $-0.16 \pm 0.03$ & 0.85 \\
\hline Oblique trefoil $Z^{3,3}$ (cornea) & $0.00 \pm 0.09$ & $-0.02 \pm 0.10$ & 0.506 & $0.01 \pm 0.11$ & $-0.01 \pm 0.09$ & 0.250 & $0.02 \pm 0.12$ & $0.00 \pm 0.12$ & 0.339 \\
\hline orizontal coma Z $Z^{3,1}$ (cornea) & $-0.01 \pm 0.15$ & $-0.03 \pm 0.15$ & 0.307 & $0.00 \pm 0.39$ & $-0.05 \pm 0.26$ & ו & $-0.04 \pm 0.40$ & -0.06 & 0.964 \\
\hline Vertical coma Z Z,-1 (Cornea) & $-0.04 \pm 0.20$ & $-0.01 \pm 0.18$ & 0.351 & $-0.66 \pm 0.23$ & $-0.39 \pm 0.23$ & $0.000^{*}$ & $-0.68 \pm 0.31$ & $-0.36 \pm 0.27$ & 0.000 \\
\hline Vertical trefoil $Z^{3,-3}$ (cornea) & $-0.07 \pm 0.12$ & $-0.07 \pm 0.12$ & 0.864 & $-0.08 \pm 0.14$ & 1.00 & 0.600 & $-0.08=$ & $-0.07 \pm 0.14$ & 0.454 \\
\hline Spherical aberration $Z^{4,0}$ (cornea) & $0.19 \pm 0.08$ & $0.20 \pm 0.07$ & 0.398 & $0.41 \pm 0.16$ & $0.26 \pm 0.12$ & $0.000^{*}$ & $0.42 \pm 0.14$ & $0.26 \pm 0.13$ & 0.000 \\
\hline
\end{tabular}

*Significant difference in HOAs 1 months postoperatively $(p<0.0167)$ (the modified Bonferroni correction, $0.05 / 3$ tests) between Group-H and Group-M; "Significant difference in HOAs 3 months postoperatively $(p<0.0167$ ) (the modified Bonferroni correction, $0.05 / 3$ tests) between Group-H and Group-M. HOAs higher-order aberrations, RMS root mean square, RMS-HOA-CF: third-order to eight-order aberrations of anterior corneal surface (cornea front, CF); RMS-HOA-cornea: third-order to eight-order aberrations of total corneal surface; RMS-HOA-CB: third-order to eight-order aberrations of posterior corneal surface (cornea back, CB)

stable. These results are consistent with those of other studies, which indicated that posterior corneal HOAs could complement corneal topography information, provide insight into the corneal biomechanical response, and provide valuable information that could be used to determine the cause of poor vision quality after corneal refractive surgery $[2,3]$.

This study revealed no significant differences in anterior cornea surface, posterior cornea surface and total corneal HOAs between high and mild to moderate myopic groups

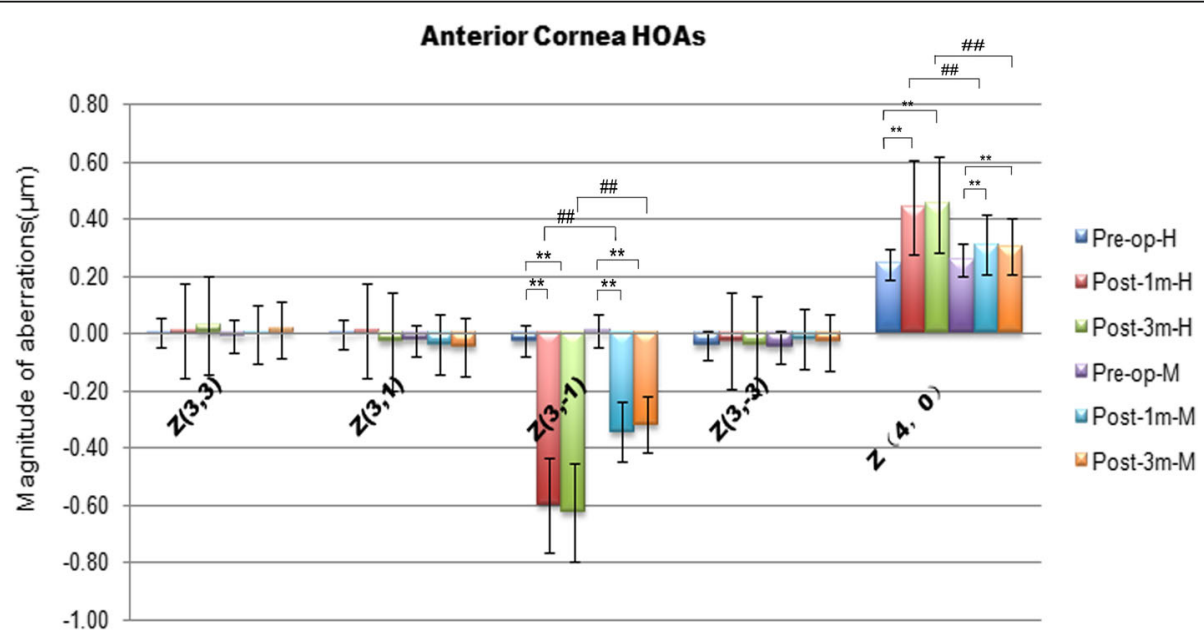

Fig. 2 Higher-order aberrations (HOAs) of the anterior cornea surface before and after SMILE surgery in Group H and group M. ${ }^{*} p,{ }^{\#} p<0.0167$ (the modified Bonferroni correction, 0.05/3 tests) 


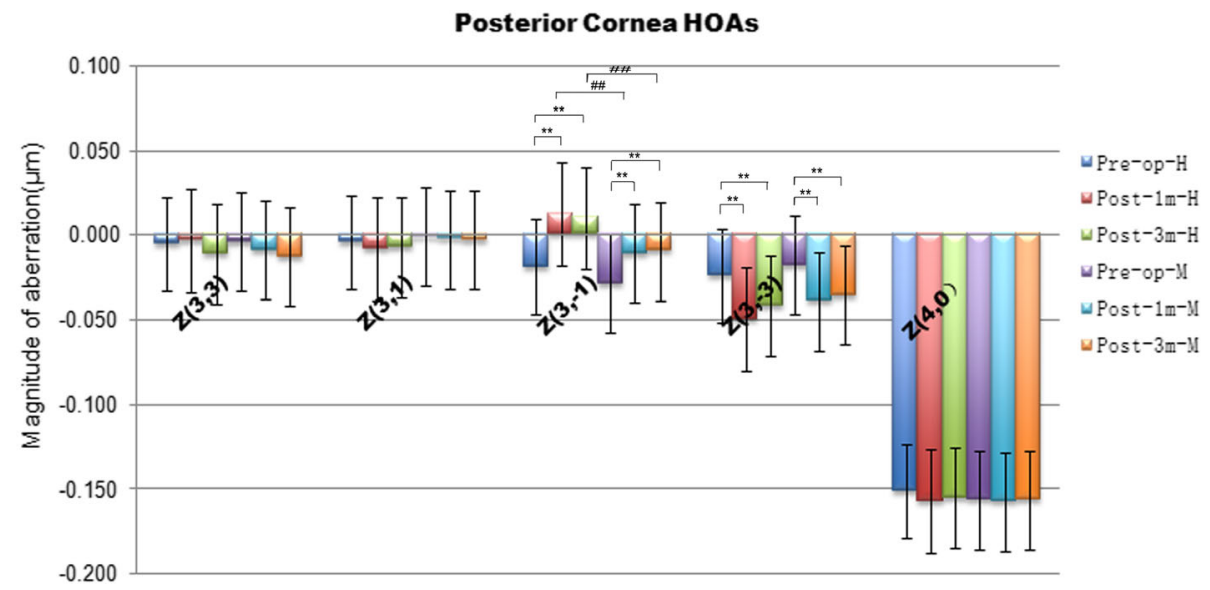

Fig. 3 Higher-order aberrations (HOAs) of the posterior cornea surface before and after SMILE surgery in Group H and group M. ${ }^{*} p,{ }^{\#} p<0.0167$ (the modified Bonferroni correction, 0.05/3 tests)

before SMILE surgery. However, significant betweengroup differences in anterior cornea surface HOAs and total corneal HOAs were detected postoperatively. In terms of SMILE-induced HOAs, there were significantly more HOAs in the high myopic group than mild to moderate group postoperatively, whereas HOAs of the posterior cornea remained almost unchanged postoperatively in both groups. The lack of significant changes in the posterior corneal surface is in agreement with that of previous studies, which examined the posterior corneal surface after PRK (Photo Refractive Keratectomy), FS-LASIK, and SMILE using Pentacam [3, 12, 13]. Wu et al. compared HOAs after SMILE, FS-LASIK, and FLEx (Femtosecond Lenticule Extraction) surgeries [3]. They reported a value of $0.19 \pm 0.03 \mu \mathrm{m}$ for third-order to eight-order aberrations of the posterior corneal surface in each surgical group postoperatively. They found that these HOAs not only remained stable postoperatively but were similar to values for HOAs obtained prior to surgery. In the present study, the number of third-order to eight-order aberrations of the posterior corneal surface in each group preoperatively and postoperatively was similar to that found by $\mathrm{Wu}$ [3]. Gyldenkerne et al. reported that the posterior corneal surface showed almost no change after FS-LASIK and SMILE procedures, which was consistent with our study [13]. Juhasz et al. analyzed changes in anterior and posterior corneal surface HOAs after PRK [12]. They found that PRK-induced HOAs increased significantly more than $76.78 \mu \mathrm{m}$ ablation depths. And the HOAs on the anterior corneal surface increased total corneal aberrations. However, posterior corneal surface HOAs remained relatively stable after surgery. They also pointed out that aberrations of the anterior corneal surface were statistically significantly higher than that of the total cornea,

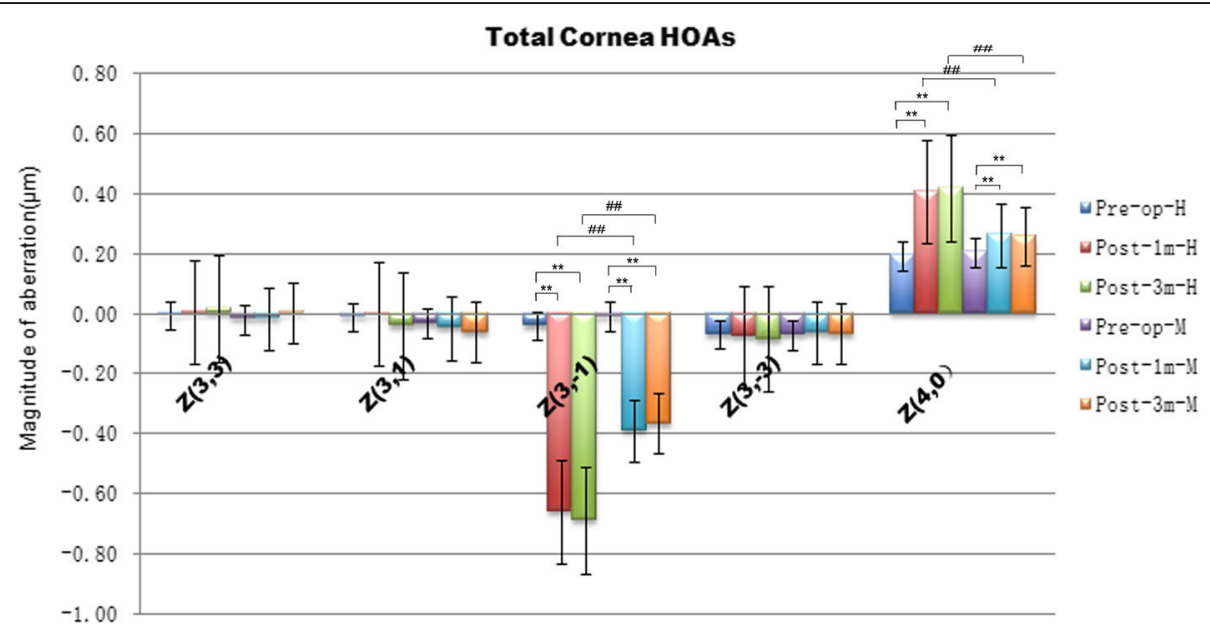

Fig. 4 Higher-order aberrations (HOAs) of the total cornea before and after SMILE surgery in Group H and group M. * $p,{ }^{\#} p<0.0167$ (the modified Bonferroni correction, 0.05/3 tests) 
Table 3 Correlations between SE and induced corneal HOAs after SMILE surgery

\begin{tabular}{|c|c|c|}
\hline Cornea aberrations & $r$ & $p$ \\
\hline RMS HOA (CF) & -0.631 & 0.000 \\
\hline RMS HOA (CB) & 0.011 & 0.867 \\
\hline RMS HOA (cornea) & -0.648 & 0.000 \\
\hline Oblique trefoil $Z^{3,3}$ (CF) & 0.047 & 0.516 \\
\hline Horizontal coma Z $Z^{3.1}$ (CF) & 0.028 & 0.692 \\
\hline Vertical coma $Z^{3-1}$ (CF) & 0.616 & 0.000 \\
\hline Vertical trefoil $Z^{3-3}$ (CF) & 0.076 & 0.290 \\
\hline Spherical aberration $Z^{4,0}$ (CF) & -0.582 & 0.000 \\
\hline Oblique trefoil $Z^{3,3}$ (CB) & -0.046 & 0.517 \\
\hline Horizontal coma Z $Z^{3,1}$ (CB) & 0.026 & 0.718 \\
\hline Vertical coma $Z^{3,-1}$ (CB) & -0.323 & 0.000 \\
\hline Vertical trefoil $Z^{3,-3}(C B)$ & -0.060 & 0.398 \\
\hline Spherical aberration $Z^{4,0}(\mathrm{CB})$ & 0.037 & 0.601 \\
\hline Oblique trefoil Z ${ }^{3,3}$ (cornea) & 0.027 & 0.702 \\
\hline Horizontal coma Z ${ }^{3,1}$ (cornea) & 0.026 & 0.715 \\
\hline Vertical coma $Z^{3,-1}$ (cornea) & 0.613 & 0.000 \\
\hline Vertical trefoil $Z^{3,-3}$ (cornea) & 0.070 & 0.329 \\
\hline Spherical aberration $Z^{4,0}$ (cornea) & 0.561 & 0.000 \\
\hline
\end{tabular}

RMS-HOA-CF: third-order to eight-order aberrations of anterior corneal surface (cornea front, CF); RMS-HOA-cornea: third-order to eight-order aberrations of total corneal surface; RMS-HOA-CB: third-order to eight-order aberrations of posterior corneal surface (cornea back, CB)

indicating that the posterior corneal surface plays a compensatory role in the balance of corneal aberrations in myopic eyes.

This study also found that spherical aberrations induced by the SMILE procedure increased in the anterior corneal surface and total cornea. The induction of spherical aberrations was significantly greater in the high myopic group than mild to moderate group. However, spherical aberrations of the posterior corneal surface remained almost unchanged in both groups after surgery. SMILE-induced coma aberrations, especially vertical coma aberrations of the anterior surface, posterior surface, and total cornea changed in both groups, with a significantly greater increase in vertical coma aberrations in the high myopic group than mild to moderate group. These results were similar to study of Wu et al. [3] Their study indicated that FS-LASIK, FLEx, and SMILE surgeries induced spherical aberrations and coma of the anterior surface and total cornea. The SMILE surgery induced fewer spherical aberrations of the anterior cornea and total cornea than FLEx procedure, and posterior corneal spherical aberrations significantly increased after FS-LASIK surgery. Thus, the SMILE surgery seems to induce fewer posterior corneal coma aberrations as compared with the FLEx surgery. Another study that compared aberrations induced by FLEx and wavefront-guided LASIK procedures indicated that FLEx induced fewer spherical aberrations but the same amount of coma aberrations as compared with that observed using wavefront-guided LASIK1 [14]. Gertnere et al. found a reduced incidence of spherical aberrations in a FLEx group but a higher incidence of induced coma aberrations in an FS-LASIK group [15]. Lin et al. compared changes in aberrations induced by FS-LASIK and SMILE surgery and found a significantly lower incidence of spherical aberrations in the SMILE procedure [16]. The aforementioned studies used different LASIK ablation techniques and different microkeratomes for cutting the LASIK flap. The use of different LASIK ablation techniques and diverse methods of flap creation may result in substantial differences in HOAs [13]. The increased number of induced aberrations observed with the FS-LASIK procedure seems to be primarily associated with the ablation of corneal tissue rather than the creation of the flap, as the flap-dependent FLEx procedure does not seem to be different from that used in SMILE [13]. In the present study, there were significantly more surgically induced aberrations in the high myopia group than mild and moderate myopia group, and more central corneal tissue was removed in the high myopia group than mild to moderate myopia group. The correlation study also indicated changes in the induction of aberrations in the two groups were related to the preoperative SE, which was consistent with the findings of the study by Chen et al. [17].

Previous studies indicated that centeration and wound healing might influence the induction of coma aberrations $[18,19]$. Li et al. demonstrated that horizontal decentration induced horizontal coma aberrations but that there appeared to be no association between the magnitude of vertical decentration and induction of vertical coma aberrations [20]. In our previous study, we reported that spherical aberrations and horizontal coma aberrations increased significantly after SMILE surgery and that the increase of spherical aberrations was higher in Group $\mathrm{H}$ than that in Group M [8]. This result was similar to findings presented by Liang et al. [21]. The difference in these results may be due to different methods used to evaluate corneal aberrations. In our previous study, we used a Hartmann-Shack WASCA aberrometer (Carl Zeiss Meditec AG, Jena, Germany). To optimize postoperative vision quality, more studies are needed to investigate the resources of the vertical coma and horizontal coma.

It is known that tear film problems that might influence the measurement of the aberrations of the anterior corneal surface, especially in dry eyes. Recently, Jung reported that total HOA RMS, coma and trefoil significantly increased at $10 \mathrm{~s}$ after blinking compared with those measured immediately after blinking in dry eye patients after LASEK (Laser Assisted Subepithelial Keratomilesusis) [22]. In the study of Elmohamady [23], they evaluated dry eye after LASIK, FS-LASIK, and SMILE. They found the mean ocular 
surface disease index (OSDI) scores were significantly elevated in all groups postoperatively but were significantly lower in the SMILE group 3 months postoperatively. The mean tear breakup time (TBUT) was significantly decreased in all groups postoperatively but was significantly higher in the SMILE group 6 months postoperatively. This result indicated the influence on dry eye was minimal after SMILE surgery. In our study, there were no significant differences in aberrations in the two groups after SMILE surgery 1 and 3 months postoperatively. This result may be due to the relatively small interference of SMILE on the tear film. Of course, long-term follow-up is still needed.

There were some limitations in this study. First, this study included 197 eyes, the two groups were not of equal size, and available data covered only 3 months. Longer term follow-up visits would have been desirable. Second, for bilaterally treated patients, there may be a correlation between the two eyes of one patient. This is a common mistake in ophthalmology research, for the overall variance of a sample of measurements combined from both eyes is likely to be an underestimate of the true variance resulting in an increased risk of a Type 1 error [24]. Future studies of the association of HOAs and corneal biomechanics with vision quality are needed to shed light on.

In conclusion, third-order to eight-order aberrations, particularly spherical aberrations and vertical coma aberrations of the anterior cornea and total cornea significantly increased after SMILE surgeries. In contrast, posterior corneal surface HOAs remained relatively unchanged. The induction of aberrations postoperatively was related to the preoperative SE. Further and larger studies, with longer-term follow-ups are needed.

\section{Abbreviations \\ CB: Posterior cornea surface/Cornea Back; CF: Anterior cornea surface/Cornea Front; FLEx: Femtosecond lenticule extraction; FS-LASIK: Femtosecond laser- assisted LASIK; HOAs: High-order aberrations; LASEK: Laser Assisted Subepithelial Keratomilesusis; LASIK: Laser-assisted in situ keratomileusis; OSDI: Ocular surface disease index; PRK: Photo Refractive Keratectomy; RMS: Root mean square; SE: Spherical equivalent; SMILE: Small incision lenticule extraction; TBUT: Tear breakup time}

\section{Acknowledgements}

None.

\section{Funding}

This study was funded by National Natural Science Foundation of China (No. 81500694), Zhejiang Province Key Research and Development Program(No. 2015C03042).

\section{Availability of data and materials}

The data have not been placed in any online data storage. The datasets generated and analyzed during the study are available upon request from the first author.

\section{Authors' contributions}

HYJ conceived of the study and drafted the manuscript. TW collected the data. YXN performed the statistical analysis. FW collected the data. KY revised the manuscript. All authors read and approved the final manuscript.

\section{Ethics approval and consent to participate}

This study followed the tenets of the Declaration of Helsinki and was approved by the ethics committee of the Second Affiliated Hospital, School of Medicine, Zhejiang University. Informed written consent was obtained from all subjects.

\section{Consent for publication}

Not applicable.

\section{Competing interests}

The authors declare that they have no competing interests.

\section{Publisher's Note}

Springer Nature remains neutral with regard to jurisdictional claims in published maps and institutional affiliations.

Received: 2 February 2018 Accepted: 30 October 2018

Published online: 12 November 2018

References

1. Li L, Cheng GPM, Ng ALK, Chan TCY, Jhanji V, Wang Y. Influence of refractive status on the higher-order aberration pattern after small incision lenticule extraction surgery. Cornea. 2017;36(8):967-72.

2. Maeda N, Nakagawa T, Kosaki R, Koh S, Saika M, Fujikado T, Nishida K. Higher-order aberrations of anterior and posterior corneal surfaces in patients with keratectasia after LASIK. Invest Ophthalmol Vis Sci. 2014;55(6):3905-11.

3. Wu W, Wang Y. Corneal higher-order aberrations of the anterior surface, posterior surface, and total cornea after SMILE, FS-LASIK, and FLEx surgeries. Eye Contact Lens. 2016:42(6):358-65.

4. Shah R, Shah S, Sengupta S. Results of small incision lenticule extraction: all-in-one femtosecond laser refractive surgery. J Cataract Refract Surg. 2011:37(1):127-37.

5. Sekundo W, Kunert KS, Blum M. Small incision corneal refractive surgery using the small incision lenticule extraction (SMILE) procedure for the correction of myopia and myopic astigmatism: results of a 6 month prospective study. Br J Ophthalmol. 2011:95(3):335-9.

6. Kim JR, Hwang HB, Mun SJ, Chung YT, Kim HS. Efficacy, predictability, and safety of small incision lenticule extraction: 6-months prospective cohort study. BMC Ophthalmol. 2014;14:117-23.

7. Reinstein DZ, Carp GI, Archer TJ, Gobbe M. Outcomes of small incision lenticule extraction (SMILE) in low myopia. J Refract Surg. 2014;30(12):812-8.

8. Jin HY, Wan T, Wu F, Yao K. Comparison of visual results and higher-order aberrations after small incision lenticule extraction (SMILE): high myopia vs. mild to moderate myopia. BMC Ophthalmol. 2017;17(1):118-25.

9. Shen Y, Chen Z, Knorz MC, Li M, Zhao J, Zhou X. Comparison of corneal deformation parameters after SMILE, LASEK, and femtosecond laser-assisted LASIK. J Refract Surg. 2014;30(5):310-8.

10. Pedersen IB, Ivarsen A, Hjortdal J. Three-year results of small incision lenticule extraction for high myopia: refractive outcomes and aberrations. J Refract Surg. 2015;31(11):719-24.

11. Tan DK, Tay WT, Chan C, Tan DT, Mehta JS. Postoperative ocular higherorder aberrations and contrast sensitivity: femtosecond lenticule extraction versus pseudo small-incision lenticule extraction. J Cataract Refract Surg. 2015:41(3):623-34.

12. Juhasz E, Kranitz K, Sandor GL, Gyenes A, Toth G, Nagy ZZ. Wavefront properties of the anterior and posterior corneal surface after photorefractive keratectomy. Cornea. 2014;33(2):172-6.

13. Gyldenkerne A, Ivarsen A, Hjortdal JØ. Comparison of corneal shape changes and aberrations induced by FS-LASIK and SMILE for myopia. J Refract Surg. 2015;31(4):223-9.

14. Kamiya K, Shimizu K, Igarashi A, Kobashi H, Komatsu M. Comparison of visual acuity, higher-order aberrations and corneal asphericity after refractive lenticule extraction and wavefront-guided laser-assisted in situ keratomileusis for myopia. Br J Ophthalmol. 2013;97:968-75.

15. Gertnere J, Solomatin I, Sekundo W. Refractive lenticule extraction (ReLEx flex) and wavefront-optimized Femto-LASIK: comparison of contrast sensitivity and high-order aberrations at 1 year. Graefes Arch Clin Exp Ophthalmol. 2013;251(5):1437-42.

16. Lin F, Xu Y, Yang Y. Comparison of the visual results after SMILE and femtosecond laser-assisted LASIK for myopia. J Refract Surg. 2014;30:248-54. 
17. Chen X, Wang Y. Comparison of ocular higher-order aberrations after SMILE and wavefront-guided femtosecond LASIK for myopia. J Ophthalmol. 2017;17(1):42.

18. Yu Y, Zhang W, Cheng X, Cai J, Chen H. Impact of Treatment Decentration on Higher-Order Aberrations after SMILE. J Ophthalmol. 2017;2017:9575723.

19. Li X, Wang $Y$, Dou R. Aberration compensation between anterior and posterior corneal surfaces after small incision lenticule extraction and femtosecond laser-assisted laser in-situ keratomileusis. Ophthalmic Physiol Opt. 2015;35:540-51.

20. Li M, Zhao J, Miao H, Shen Y, Sun L, Tian M, Wadium E, Zhou X. Mild decentration measured by a Scheimpflug camera and its impact on vision quality following SMILE in the early learning curve. Invest Ophthalmol Vis Sci. 2014;55(6):3886-92

21. Liang G, Zha X, Zhang F. Analysis of higher-order aberrations ( $\mathrm{HOAs}$ ) and related factors after small incision lenticule extraction (SMILE) surgery. Clin J Optom Ophthalmol Vis Sci. 2015;17(11):644-8.

22. Jung HH, YS J, Oh HJ, Yoon KC. Higher order aberrations of the corneal surface after laser subepithelial keratomileusis. Korean J Ophthalmol. 2014;28(4):285-91.

23. Elmohamady MN, Abdelghaffar W, Daifalla A, Salem T. Evaluation of femtosecond laser in flap and cap creation in corneal refractive surgery for myopia: a 3-year follow-up. Clin Ophthalmol. 2018;12:935-42.

24. Armstrong RA. Statistical guidelines for the analysis of data obtained from one or both eyes. Ophthalmic Physiol Opt. 2013;33:7-14.

Ready to submit your research? Choose BMC and benefit from:

- fast, convenient online submission

- thorough peer review by experienced researchers in your field

- rapid publication on acceptance

- support for research data, including large and complex data types

- gold Open Access which fosters wider collaboration and increased citations

- maximum visibility for your research: over $100 \mathrm{M}$ website views per year

At $\mathrm{BMC}$, research is always in progress.

Learn more biomedcentral.com/submissions 